\title{
Evaluation of critical blockage ratio and pulse length in a pulse detonation engine using CFD and MATLAB
}

\author{
S. Srikrishnan ${ }^{1, *}$, P. K. Dash ${ }^{2}$, and V. Jayakumar ${ }^{3}$ \\ ${ }^{1}$ Mechanical Department, IARE, Hyderabad, India \\ ${ }^{2}$ Aeronautical Department, MLR Institute of Technology, Hyderabad, India \\ ${ }^{3}$ Mechanical Department, Saveetha School of Engineering, Saveetha Institute of Medical and Technical Sciences, Chennai, India
}

\begin{abstract}
A Pulse Detonation Engine (PDE) is a new invented propulsion device that takes advantage of the pressure rise inherent to the efficient burning of fuel-air mixtures via detonations. Detonation initiation is a critical process that occurs in the cycle of a PDE. A practical method of detonation initiation is Deflagration-to-Detonation Transition (DDT), which describes the acceleration of a subsonic deflagration created using low initiation energies to a supersonic detonation. The DDT process is not well understood due to a wide range of time and length scales involving complex chemistry, turbulence and unsteady pressure waves. This paper discuss about the effects of blocking ratio in the augmentation of detonation pressure and velocity inside a cylindrical tube of diameter $0.0254 \mathrm{~m}$ and a length of $1 \mathrm{~m}$. The blockages are rectangular in shape placed at $2 / 3^{\text {rd }}$ distances of the length of the tube and the heights of the blockages are varied in terms of the diameter of the tube as $1 / 4^{\text {th }}, 1 / 3^{\text {rd }}, 1 / 2,2 / 3^{\text {rd }}$ and $3 / 4^{\text {th }}$ the diameter of the tube. The setup is then analyzed in MATLAB using the physics of Friedlander's equation, which formulate the decay time duration of pressure across the tube length, with and without the blockage. Further, a 2D CFD analysis through ANSYS Workbench is conducted which gave the effective blocking ratio in a rectangular type of blockage placed at the $2 / 3^{\text {rd }}$ position of the length of the tube and the results are compared. For variable pressures ranging from $1 \mathrm{MPa}$ to $100 \mathrm{MPa}$ input, the effective pulse length is around 0.25 seconds after which the decay of pressure and temperature attain the critical limit. Also it is found that the maximum feasible velocity occurs for an inlet pressure of $10 \mathrm{MPa}$ and $2 / 3^{\text {rd }}$ height of the blockage where the corresponding outlet velocity is $4692 \mathrm{~m} / \mathrm{s}$ and outlet total pressure being $10.542 \mathrm{MPa}$.
\end{abstract}

\section{Introduction}

The new era of detonation of air-breathing propulsion has conceived from the studies on fuel energy transformation in stabilized oblique detonation waves. The technology gradually immerged for investigations and practical development of propulsion engines operating on propagating detonations in a pulse mode [1]. Contrary to the oblique-detonation concept that is applicable to hypersonic flight at velocities comparable or higher than the Chapman-Jouguet (CJ) detonation velocity of the fuel-air mixture, the concept of pulse detonation engine (PDE) is attractive for both subsonic and supersonic flight with PDE as a main propulsion unit or as an afterburner in turbojet or turbofan propulsion system. In particular, PDE [2] based propulsion is attractive for flight Mach number up to about 3 to 4 .

In a PDE, detonation is initiated in a tube that serves as the combustor [3]. The detonation wave rapidly traverses the chamber resulting in a nearly constant-volume heat addition process that produces a high pressure in the combustor and provides the thrust [4]. The operation of
PDE configurations at high detonation-initiation frequency (about $100 \mathrm{~Hz}$ and over) [5, 7] can produce a near-constant thrust. In general, the near-constantvolume operational cycle of PDE provides a higher thermodynamic efficiency as compared to the conventional constant-pressure (Brayton) cycle used in gas turbines and ramjets [6]. The advantages of PDE for air-breathing propulsion are simplicity and easy scaling, reduced fuel consumption, and intrinsic capability of operation from zero approach stream velocity to high supersonic flight speeds [8].

The global interest in the development of PDE [9] for propulsion has led to numerous studies on detonations, particularly pertaining to its control and confinement. This is evident from the formation of collaborative teams by universities and industry worldwide [10].

Several reviews have been already presented at various meetings [1-10] and published in archival journals [1126]. Wedge- and cone-induced oblique shock/detonation waves have received widespread attention due to their potential application in hypersonic propulsion devices. 
These include ram accelerators and oblique detonation wave engines (ODWEs). Experimental observations of oblique shock-induced detonation, such as those in have shown that at least two different types of flow can arise. The first is the direct initiation of a detonation wave within a stagnation region. The second is an oblique shock wave that transitions to an oblique detonation wave a certain distance from the wedge's leading edge. Numerical simulation of wedge- and cone-induced detonation has been done recently. The above-cited studies were concerned with the body in a semi-infinite domain. Moreover, the interaction is induced by a long wedge, that is, one where the shoulder does not affect the structure of the interaction zone, an exception being. Generally, the numerical studies showed two possible ways that a detonation is achieved. In the first, an attached oblique shock in the incoming premixed combustible mixture transitions to a detonation, arising from the compression. In the second, a direct initiation is obtained.

From cited research inactivation the PDE advantages mentioned above, a number of challenging fundamental and engineering problems has yet to be solved in order to use propagating detonations for propulsion and realize. These problems deal basically with low cost achievement and control of successive detonations in a propulsion device. To ensure rapid development of a detonation wave within a short cycle time, one needs to apply (1) efficient liquid fuel injection and air supply systems to provide fast and nearly homogeneous mixing of the components in the detonation chamber (DC); (2) low energy source for detonation initiation to provide fast and reliable detonation onset; (3) cooling technique for rapid, preferably recuperative, heat removal from the walls of DC to ensure stable operation and avoid premature ignition of FAM leading to detonation failure; (4) geometry of the combustion chamber to promote detonation initiation and propagation at lowest possible pressure loss and to ensure high operation frequency; and (5) control methodology that allows for adaptive, active control of the operation process to ensure optimal performance at variable flight conditions, while maintaining margin of stability. In addition to the fundamental issues dealing with the processes in the DC, there are other issues such as (6) efficient integration of PDE with inlets and nozzles to provide high performance; and (7) efficient coupling of DCs in a multi tube PDE configuration. Among the most challenging engineering issues, is the problem of durability of the propulsion system. As the structural components of PDE are subject to repeated highfrequency shock loading and thermal deformations, a considerable wear and tear can be expected within a relatively short period of operation. The other problem is noise and vibration.

The previous paper analysed the effects of different types of blockages like triangular, semi-circular and rectangular in $1 / 3^{\text {rd }}, 1 / 2$ and $2 / 3^{\text {rd }}$ positions of the length of the tube of diameter $0.0124 \mathrm{~m}$ and $0.25 \mathrm{~m}$ length thus finding that the rectangular blockage is the most feasible when placed at the $2 / 3^{\text {rd }}$ distance of the tube [27]. This paper presents the effects of blocking ratio in the augmentation of detonation pressure and velocity inside a cylindrical tube of diameter $0.0254 \mathrm{~m}$ and a length of 1 $\mathrm{m}$. The blockages are rectangular in shape placed at $2 / 3^{\text {rd }}$ distance of the length of the tube and the heights of the blockages are also varied in terms of the diameter of the tube as $1 / 4^{\text {th }}, 1 / 3^{\text {rd }}, 1 / 2,2 / 3^{\text {rd }}$ and $3 / 4^{\text {th }}$ of the diameter of the tube. A 2D CFD analysis through ANSYS Workbench is conducted which gave the effective blocking ratio in a rectangular type of blockage placed at the $2 / 3$ rd position of the length of the tube. Only the height of the blockages is varied and the results are compared. The setup is then analysed in MATLAB using the physics of Friedlander's equation, which formulate the decay time duration of pressure across the tube length, with and without the blockage.

For variable pressures ranging from $1 \mathrm{MPa}$ to $100 \mathrm{MPa}$ input, the effective pulse length is around 0.25 seconds after which the decay of pressure and temperature attain the critical limit. This result gives a predictive analysis of detonation wherein the frequency of spark for inducing controlled combustion inside the tube can be studied.

\section{Methodology}

\subsection{Computational Method}

In this study, a commercial code, 'FLUENT' which is based on the Navier-Stokes equation is utilized for this analysis. 'GAMBIT' which is the pre-processor of this study has a 'Journaling Function' for auto-grid generation. This function is useful to mesh the similar types of models repeatedly [6]. 'Realizable $\mathrm{k}-\varepsilon$ ' is selected as a turbulence model, which is a variant of the 'Standard $\mathrm{k}-\varepsilon$ ' model. Among the two equation Reynolds Average Navier-Stokes (RANS) turbulence models, 'Realizable $\mathrm{k}-\varepsilon$ ' is known as the most suitable for a turbulent flow.

\subsection{Modelling}

It has been studied in previous paper that the rectangular type of blockage introduced in the $2 / 3$ rd position of the tube length would have the maximum detonation effect. Using the results, the current work is done to establish the critical blockage ratio to find out the most feasible height of the blockage to induce detonation in a closed pipe of diameter $0.0253 \mathrm{~m}$ and a length of $1 \mathrm{~m}$. The modelling has been done in GAMBIT, where a $1 \mathrm{~m}$ length of pipe is taken with blockages being constructed at $2 / 3 \mathrm{rd}$ distance along the length of the tube. The blockage heights have been varied as 1/4th, 1/3rd, 1/2, $2 / 3 \mathrm{rd}, 3 / 4$ th of the diameter of the tube. 


\subsection{Meshing}

The scaled model was meshed using GAMBIT. The length of the tube was taken as the scale of the element size. The element size was taken to be 0.049 and a quadtri type of mesh was selected to mesh the $2 \mathrm{D}$ faces after the extrusion of the blockages placed at the $2 / 3$ rd the distance from the origin. A total of 5 different meshes were created for the effective study of the critical blockage ratio. A sample of the mesh has been shown in Fig. 1

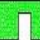

Fig. 1. Mesh created for $1 \mathrm{~m}$ length and $2.53 \mathrm{~cm}$ diameter tube with blockage placed at $2 / 3 \mathrm{rd}$ distance along the length of the tube.

\subsection{Boundary Conditions}

The listed meshes generated using GAMBIT was then imported into ANSYS 16.0 as case files. Multiphase analysis of a kerosene air vapour mixture has been done on selected mesh files to arrive at the final conclusion. Uniform pressure growth varying from $1 \mathrm{MPa}$ to 1000 MPa was adapted and a coupled condition of pressure and velocity has been created at inlet. Velocity at inlet was set a uniform $0.15 \mathrm{~m} / \mathrm{s}$ derived from combustion velocity of kerosene air vapour combustion data (CHEMKIN). All the variation of velocity during analysis is counted in the top and bottom wall boundaries inclusive of the blockage as shown in the Fig. 1. Data computing is carried out at outflow boundary of tube and considered as a diaphragm.

\subsection{Pre-processing}

The model for solving the $2 \mathrm{D}$ analysis was considered as a k-e model without viscous heating of the surface entities to minimize the data loss of temperature rise at the surface of the tube. A multiphase analysis with primary kerosene vapour and secondary air was considered to be flowing through the inlet with an ambient velocity of $0.15 \mathrm{~m} / \mathrm{s}$ and ignition temperature of $1500 \mathrm{~K}$ was done on the $2 \mathrm{D}$ model. Initial operating conditions were for a nominal pressure of $1 \mathrm{MPa}$ and linear increment of pressure was considered for evening out the acquired data to imply optimum pressure considered for achieving detonation transiting from deflagration of the mixture. The analysis was done in transient condition with a time step size of 0.01 for the duration of $10 \mathrm{~s}$.

\subsection{MATLAB}

In the calculation and presentation of the pressure blast profiles, numerous methods have been proposed. Baker reports that modified Friedlander's equation given by:

$$
P(t)=P_{o}+P_{s}\left(I-\left(t / T^{+}\right)\right)^{-b t / T^{+}}
$$

Where again $t$ is measured from the time of arrival, Ps + is the peak side-on overpressure $\mathrm{P}_{0}$ is the ambient atmospheric pressure, and $b$ is the decay coefficient. Thus, a curve fitting of experimental data may be done by matching any three of four blast characteristics: peak side-on overpressure, $\mathrm{P}_{\mathrm{s}}+$, positive phase impulse, $\mathrm{I}^{+}$, positive phase duration, $\mathrm{T}^{+}$. The peak overpressure is the highest pressure the initial blast wave creates over the ambient atmospheric pressure. The positive phase impulse is the area under the pressure curve for the duration of the time period of positive overpressure, that same time period is the positive Phase duration, and the initial decay rate is a measure of how quickly the Pressure returns from the spike of peak over pressure to the ambient pressure.

As the pressure profile is of a decaying nature, pressure decay w.r.t range is presented using the following formula

$$
P(R)=P_{o}+P_{s} e^{-\gamma R}
$$

$P_{s}$ is the peak side-on overpressure, $\gamma$ is the damping ratio, $P_{0}$ is the ambient pressure, $R$ is distance in the space, and $\mathrm{P}(\mathrm{R})$ is the pressure with respect space again where $t$ is measured from the time of arrival, ta. Thus, this simplified form can be altered to fit the desired profile curve. Negative pressure phase is ignored in this study. Find only one proposed functional form for this phase from the works of Brode (1955):

$$
P(t)=P_{o}+P_{s}(t / T)(I-(t / T))^{-b t / T+}
$$

Where Po is the ambient pressure, Ps is the peak negative phase overpressure, $\mathrm{T}^{-}$is the duration of the negative phase, and $t$ is measured from the end of the positive phase $\left(t_{0}+T\right)$. As detonations can vary in both composition and manner of destruction forces, they are usually comported by the shattering effect of sudden release of energy of each detonations or their blast pressure.

A MATLAB coding is made to study the effects of blast waves inside a tube $1 \mathrm{~m}$ long with a diameter of $2.53 \mathrm{~cm}$ with placing blockages at the various locations through the length of the tube. The study is done to calculate the peak pressure of detonation and as well as to study the decay time of pressure during the flow through the tube to determine the effective pulse length in a pulse detonating engine. A sample graph of the peak pressure built up and decay of blast wave is shown in Fig 2 . 


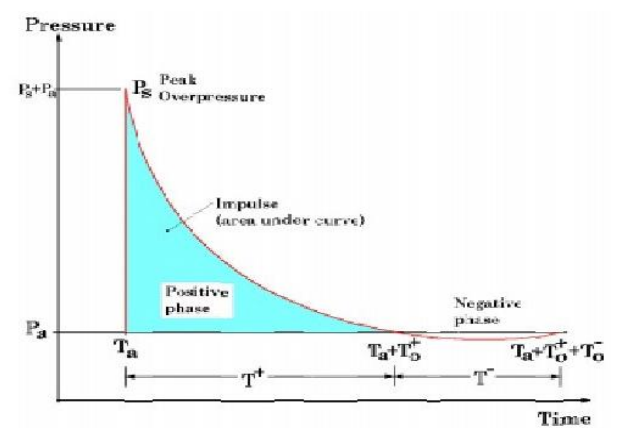

Fig. 2. Canonical blast profile using Friedlander's equation

\section{Result and discussions}

\subsection{Formation of DDT}

Deflagration-to-detonation transition (DDT) is the process by which an initially laminar flame undergoes a sequence of changes in propagation mechanism, ultimately resulting in a self-sustained supersonic detonation. While DDT is typically thought of as an acceleration process (since the combustion wave velocity can span four orders of magnitude), it is more properly considered as a process of altering the mechanism of wave propagation, with the propagation velocity reflecting the rate at which different mechanisms occur. Appreciating when these different mechanisms are at work lays a foundation for understanding and ultimately controlling DDT. The initial stages of flame kernel formation and growth as a laminar flame are dominated by diffusion of heat and radicals to the unburned layer ahead of the flame. A whole host of natural instability mechanisms act to wrinkle the flame and increase the burning rate per unit volume and, consequently, increase the propagation velocity.

\subsection{Turbulent flow}

A more significant role is played by the flow created ahead of the flame due to volumetric dilatation of the combustion products. In a pipe this flow becomes turbulent and a flame propagating into a turbulent flow field undergoes a dramatic increase in the burning rate. This transition to turbulence also sets the stage for feedback between the flow ahead of the flame and the flame itself. The feedback can occur via a fluid dynamic mechanism, whereby an increase in burning rate increases the flow velocity of the gas ahead of the flame, thus amplifying the intensity of turbulent flow into which the flame propagates. The unsteady flow ahead of the turbulent flame also allows for a gas dynamic feedback mechanism, provided the unsteady compression waves sent ahead of the flame increase the temperature sufficiently to produce an accelerating effect on the reaction rates. Regardless of the particular mechanism, when the combustion wave reaches very high turbulent flame speeds $(600-1000 \mathrm{~m} / \mathrm{s})$, it clearly becomes a mixing limited, rather than a diffusion controlled, process. The fact that high-velocity turbulent flames propagate with mixing rates apparently greater than those required to quench the flame. When the turbulent flame reaches velocities $(\sim 1000 \mathrm{~m} / \mathrm{s})$ where the compression waves pushed ahead of the flame coalesce into a shock generating sufficient adiabatic compression to initiate chemical reactions, the conditions for the final onset of detonation may be present. The final initiation of detonation is usually associated with the abrupt appearance of explosion centers or "hot spots" in the shock - turbulent flame complex (the so-called "explosion in the explosion") which rapidly accelerate in the shocked gas to merge with the initial SHOCK.

Another mode of DDT due to the progressive amplification of pressure waves traversing the reaction zone at the final stages of initiation forms the detonation wave. It appears that the onset of detonation invariably involves a rapid wave amplification process. The result of this amplification process is an initially overdriven detonation, often with wave speeds exceeding the steady Chapman-Jouguet (CJ) wave speed by $50-100 \%$, which quickly decays to a steadily propagating detonation. The observations made due to the position of the blockage placed at the $2 / 3^{\text {rd }}$ position of the length of the tube with the variation of height of the blockage with reference to the inlet pressure the corresponding pressure and velocity at the outlet of the tube have been shown in Table 1 and Table 2.

Table 1. Variation of outlet velocity with reference to the position of the blockage and inlet pressure.

\begin{tabular}{|c|c|c|c|c|c|c|}
\hline \multirow{3}{*}{$\begin{array}{c}\text { Pressure } \\
\text { MPA }\end{array}$} & \multirow{3}{*}{$\begin{array}{c}\text { INLET } \\
\text { VELOCITY } \\
\mathrm{m} / \mathrm{s}\end{array}$} & \multicolumn{5}{|c|}{ VELOCITY AT OUTLET m/s } \\
\hline & & \multicolumn{5}{|c|}{ HEIGHT OF BLOCKAGE } \\
\hline & & $\begin{array}{c}0.632 \\
\mathrm{~cm}\end{array}$ & $\begin{array}{c}0.843 \\
\mathrm{~cm}\end{array}$ & $\begin{array}{c}1.265 \\
\mathrm{~cm}\end{array}$ & $\begin{array}{c}1.686 \\
\mathrm{~cm}\end{array}$ & $\begin{array}{c}1.897 \\
\mathrm{~cm}\end{array}$ \\
\hline 1 & 0.15 & 1425 & 1400 & 1433 & 1477 & 1223 \\
\hline 10 & 0.15 & 4572 & 4674 & 4548 & 4692 & 3867 \\
\hline 100 & 0.15 & 15244 & 14751 & 14143 & 14650 & - \\
\hline
\end{tabular}

Table 2. Variation of outlet pressure with reference to the position of the blockage and inlet pressure.

\begin{tabular}{|c|c|c|c|c|c|}
\hline \multirow{3}{*}{$\begin{array}{c}\text { Pressure } \\
\text { MPA }\end{array}$} & \multicolumn{5}{|c|}{ PRESSURE AT OUTLET MPA } \\
\cline { 2 - 6 } & $\begin{array}{c}0.632 \\
\mathrm{~cm}\end{array}$ & $\begin{array}{c}0.843 \\
\mathrm{~cm}\end{array}$ & $\begin{array}{c}1.265 \\
\mathrm{~cm}\end{array}$ & $\begin{array}{c}1.686 \\
\mathrm{~cm}\end{array}$ & $\begin{array}{c}1.897 \\
\mathrm{~cm}\end{array}$ \\
\hline 1 & 1.017 & 1.013 & 1.043 & 1.05 & 1.07 \\
\hline 10 & 10.311 & 10.171 & 10.479 & 10.542 & 10.736 \\
\hline 100 & 110.5 & 101.86 & 105.273 & 105.55 & - \\
\hline
\end{tabular}

It is observed in the Table 1, 2 that, for the incremental schema of inlet pressure the variation of velocity and pressure at the exit of the tube is almost linear in nature. With regards to the exit velocity the maximum can be seen using a rectangular type of blockage placed at the $2 / 3^{\text {rd }}$ position of the tube with $1 / 3^{\text {rd }}$ the diameter of the tube as the blockage height. But this occurs in a high inlet pressure of $100 \mathrm{MPa}$ and further there is a decrement curve for all other blockage heights at the same pressure input due to the condition of choking and reverse flow scenario can be predicted. So the most feasible height can be considered to be $2 / 3^{\text {rd }}$ height of the diameter of the tube placed in the $2 / 3^{\text {rd }}$ distance of the 
length of the tube, where at the nominal input pressure of $10 \mathrm{MPa}$, it is seen a sharp increment of output velocity and pressure. Previous experimental study shows that around $40 \%$ block of the tube diameter gives the onset of DDT and gives better performance and ignition characteristics. The current comparative analysis has shown a blockage ration of $66 \%$ of the total diameter of the tube may be the most optimum of all. The following graphs (Fig. 3, 4) shows the variation of pressure and velocity at the outlet of the tube with reference to Table 1 and 2.

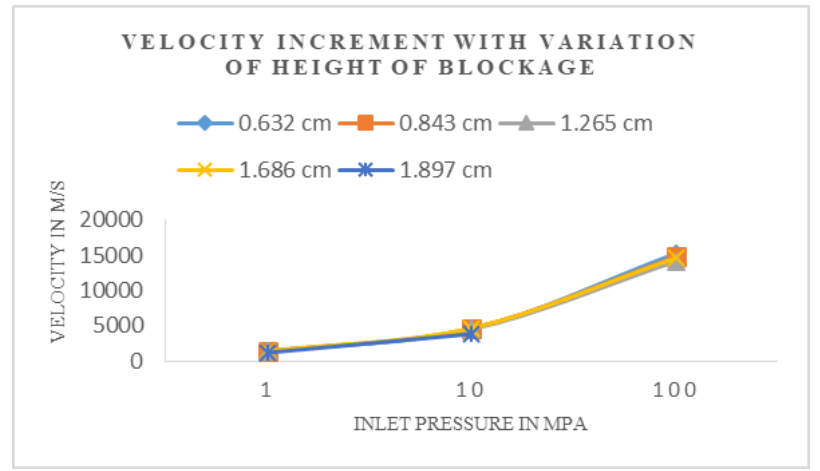

Fig. 3. Graphical representation of velocity at the outlet with variation of pressure and height

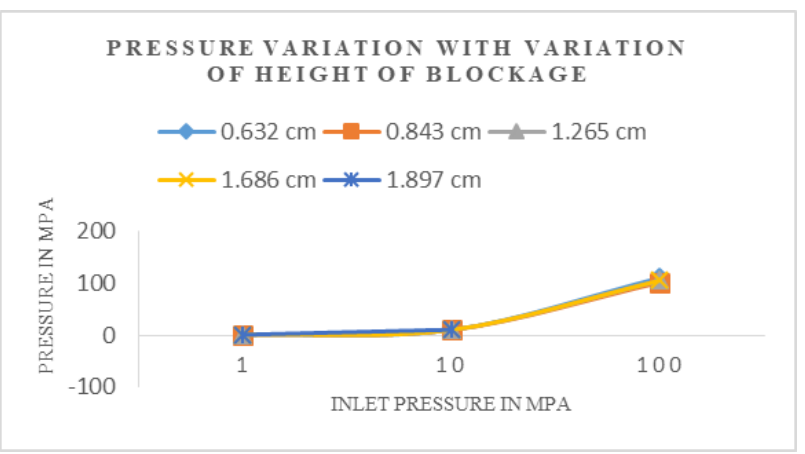

Fig. 4. Graphical representation of pressure at the outlet with variation of pressure and height

The MatLab code is generated as a combination of Friedlander's equation and the effect of peak pressure and the time of decay are studied. The variation of pressure, temperature and velocity of the flow in a tube of length $1 \mathrm{~m}$ and diameter of $0.0254 \mathrm{~m}$ with blockage kept at the $2 / 3^{\text {rd }}$ distance is analysed and the results are plotted for the same with reference to time in Fig. $5-7$.

The graphs shown above shows that the effective decay time is around 0.25 seconds from the initiation of the detonation, i.e., the pulse length for a PDE can be assumed to be around 0.25 seconds wherein the next cycle can be started. The study also shows that a maximum of $8000 \mathrm{~m} / \mathrm{s}$ of velocity may decay up to 2000 $\mathrm{m} / \mathrm{s}$ in the duration of 0.28 seconds. It can be further said that the effective pulse length may be optimized by the above result by designing a system to supply the air fuel mixture to the PDE at a differential pulse setting of 0.25 -0.28 seconds for continuous thrust.

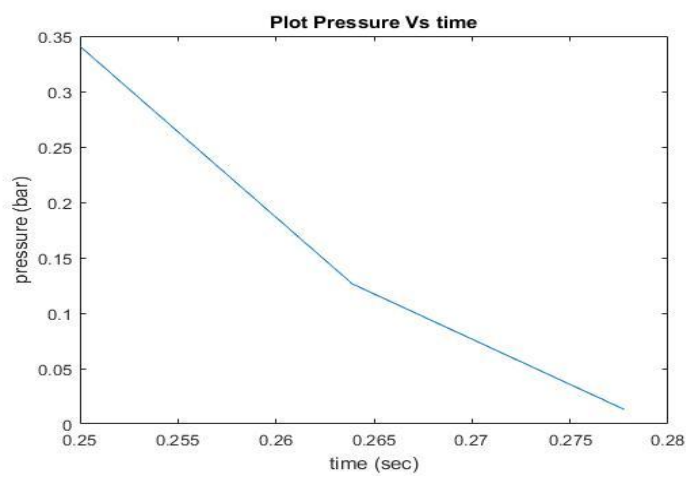

Fig. 5. Variation of pressure with reference to time

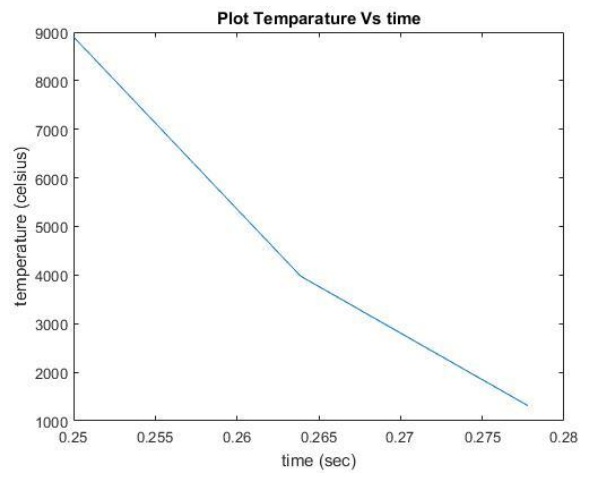

Fig. 6. Variation of temperature with reference to time

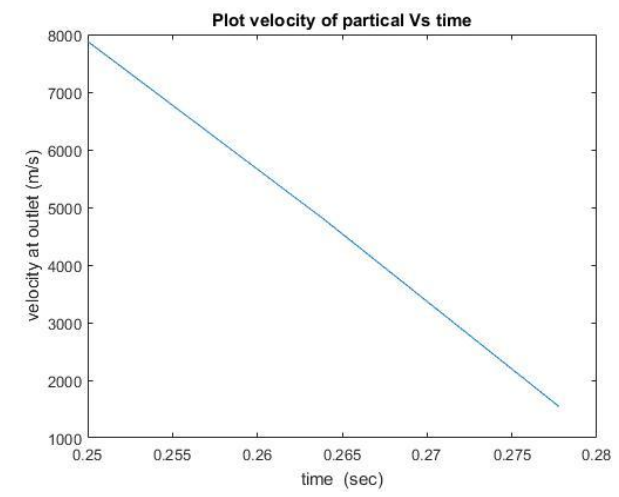

Fig. 7. Variation of velocity of particle with reference to time

\subsection{Presence of obstacle}

Without obstacle the run-up distance to detonation can be an order of magnitude larger and significantly more stochastic, due to increased sensitivity to the tube wall roughness, position of the igniter, etc. An obstacle blockage ratio of $\sim 60 \%$ and an obstacle spacing of roughly one tube diameter as being near the point of diminishing return in obstacle spacing and blockage. Thus, the results obtained with these obstacles are believed to be the near minimum length scales required for DDT, and further optimization of the obstacles 
wo9uld result in only minor changes in the length required for detonation formation. Due to the abrupt appearance of detonation at the final stage of the flame acceleration process, initiation is traditionally considered as a unique and well defined

event. Recent research on quasi-detonation, however, suggests that in tubes with obstacles, the same mechanisms responsible for DDT (local explosion centers resulting from shock interactions with obstacles) may also permit steady propagation velocities as low as one half of the CJ velocity. Thus, a CJ detonation is merely the limiting case of a continuous spectrum of combustion wave velocities, and the division between detonation and highly turbulent flame becomes indistinct. If a combustion wave is "denied" the mechanisms of detonative combustion, either by using a tube smaller than the characteristic cell size of the mixture or by using a tube with acoustic absorbing walls to dampen out cellular structure, then the wave will propagate at speeds of the order of one

half the CJ detonation velocity.

Direct initiation (i.e., near-instantaneous deposition of energy into the gaseous mixture, generating a blast wave of sufficient strength and intensity to decay to a selfsustained detonation) would require charges on the order of $\sim 100 \mathrm{~g}$ of high explosive for each cycle of initiation. Other initiation sources (sparks, lasers, etc.) cannot easily provide this amount of energy $(\sim 100-1000 \mathrm{~kJ})$ on a cyclic basis. This leaves "self-initiation," or initiation using the chemical energy of the explosive medium itself, to create the conditions necessary for the onset of detonation. The most recent estimates of the critical kernel size for planar initiation $[9,10]$ suggest that the chemical energy available within one to two cell lengths of the explosive mixture is sufficient to initiate detonation in that mixture. The usual path of selfinitiation, however, is DDT, and this typically requires an order of magnitude longer length scale before the onset of detonation occurs. For a higher hydrocarbon fuel in air (where the detonation cell size A $« 5 \mathrm{~cm}$ ), the tube diameter must be greater than A for self-sustained propagation, and typically 10 tube diameters or more are required for DDT. Thus, for hydrocarbon-air mixtures, the length required for DDT approaches or even exceeds the length scales of interest for PDE applications. The fact that, in principle, there exists sufficient chemical energy in the mixture to undergo self-initiation within one or two tube diameters suggests that a significant reduction in initiation length scales could be realized if the appropriate route to detonative combustion can be identified. The matlab code run for the program has shown the distance achievement of detonation wave and sustainability of the detonation wave. Knowledge of the mechanisms responsible for DDT and the transition between those different mechanisms is not sufficient at present to identify a "soft" initiation scheme that uses the weak igniter of DDT yet initiates on the short length scales of direct initiation. Experiments with turbulent jet initiation suggest a direction that may prove fruitful.

\subsection{Laminar to turbulent transition}

The role of an ignition in DDT is to create an initial flame kernel that will grow as a laminar flame. In confined tubes with ignition occurring at a closed end, the laminar flame will quickly become turbulent due to the various instability mechanisms discussed above. Since, for a fuel-air mixture, the laminar flame propagates at speeds of $30-50 \mathrm{~cm} / \mathrm{s}$, the time required for the flame to reach the tube wall and make the transition to a turbulent flame can comprise the majority of the time required for the entire DDT process. If a sufficiently powerful igniter is used, it may be possible to bypass the laminar to turbulent flame transition process entirely.

With ignition the flame is replaced with a small distance coverage in which the combustion products jet outward, the time required for DDT is reduced by a factor of more than two as shown in detonation length vs. $t$ diagram (Fig $5-7$ ).

Viewed as a velocity-distance $(V-x)$ diagram, however, the results obtained with the different detonation transition and sustainability for detonation wave are indistinguishable. This is because, while the ignition chamber succeeds in creating a turbulent flame, the turbulent flame must still propagate the same distance before reaching velocities sufficient for the onset of detonative combustion. While the laminar to turbulent flame transition comprises the majority of the time required for DDT, it is negligible in the length scale required for DDT. While it may be argued that a very powerful igniter, or array of igniters, may be capable of near-direct initiation of detonation, studies of detonation initiation by turbulent flames emerging from obstacleladen tubes and suggest that turbulent flame velocities in the $700-1000 \mathrm{~m} / \mathrm{s}$ range are necessary for detonation initiation, with turbulent fluctuating velocities on the order of the sonic speed. This would necessitate an initiator of sonic or supersonic jets of hot gas over the entire tube area. Such an initiator would likely consist of a volume of combustible gas that must itself undergo transition to turbulent flame propagation anyway.

The length scale required for further acceleration and transition to detonation is still very large. Thus, a powerful igniter can play an important role in rapid initiation techniques, but only as a means of initially creating a highly turbulent flame. The acceleration of that flame to detonation still consumes a considerable distance. This may be capable of direct initiation upon emerging from a tube diameter smaller than the critical diameter for detonation transmission.

\subsection{Sensitivity of mixture}

While chemical kinetics plays a role in laminar and turbulent flame propagation, it is only with the shockinitiated reactions of the final onset of detonation that the exponential nature of kinetic rates dominates the propagation mechanism as shown through matlab codes. Compared to laminar-turbulent flame transition and the acceleration of turbulent flames, chemical sensitization 
or desensitization of combustible gases for detonability has received comparatively little attention to increase in the length required for transition to detonation.

As DDT is an extremely complex phenomenon, incorporating numerous different mechanisms and orders of magnitude variations in propagation velocity, it is essential that studies of DDT carefully isolate the effect being examined. The results reviewed in this paper showed that the effect of a block size and detonation sustainability for fuel-air mixtures is confined to promoting a more rapid transition from laminar to turbulent flame and does not significantly influence the run-up distance required for detonation. Only with a turbulent jet igniter of very large diameter $(d>10 \mathrm{~A})$ can the intensities and scales of turbulence necessary for the direct onset of detonation be realized. Obstacles on the tube wall can increase the intensity of turbulence in the flame and introduce transverse pressure fluctuations (shocks), and thus dramatically increase the burning rate and strength of compression waves sent ahead of the flame. This sets the stage for shock-induced combustion and the onset of detonation by the formation of explosion centers, but detonation will not occur if the tube is smaller than the characteristic detonation length scale (the cell size). It is in this final onset of detonation that kinetic rates dominate the transition mechanism and the presence of free radicals or other chemical sensitizers can have a pronounced effect. An efficacious sensitizer for hydrocarbon fuel-air mixtures has yet to be identified, in which a heavy hydrocarbon fuel is reformed via a partial oxidation process to more sensitive hydrocarbons and free radicals, appears to be a promising way to dramatically reduce the transition length to detonation. In light of the fact that DDT is an ensemble of various mechanisms, the results reported in this paper represent the same shortcomings as prior work in DDT: examining the run-up distance to detonation alone does not isolate the various mechanisms involved. Rather, future work on DDT should be focused on defining the basic scaling laws for DDT, and then relating these laws to more fundamental parameters, such as the ratio of mixture cell size to tube diameter.

\section{Conclusions}

Based on the 2D CFD analysis and MatLab coding done for the tube of length $1 \mathrm{~m}$ and diameter $0.0254 \mathrm{~m}$ with blockages setup at the $2 / 3^{\text {rd }}$ distance of the length of tube and by varying the height of the blockages, the following conclusions have been drawn:

1. It has been observed that for a maximum pressure of $100 \mathrm{MPa}$ and $1 / 3^{\text {rd }}$ height of the blockage the velocity at the exit is maximum at $15244 \mathrm{~m} / \mathrm{s}$, which may not be feasible for the actual working process.

2. For an inlet pressure of $10 \mathrm{MPa}$ and $2 / 3^{\text {rd }}$ height of the blockage, the velocity at the exit of the tube is found to be around $4692 \mathrm{~m} / \mathrm{s}$, which is the maximum feasible velocity. The corresponding pressure at the exit is found to be $10.542 \mathrm{MPa}$.

3. The critical blockage ratio can be found around $2 / 3^{\text {rd }}$ height of diameter of the tube which has a steady incremental velocity schema for various pressure inputs.

4. The MatLab coding data concludes that the time taken for the inlet pressure to decay is around 0.275 seconds, so it can be incepted that 0.25 seconds may be the effective pulse length for a PDE.

The comparative study of both computational and MatLab coding evolves that the critical blockage impacts the exit velocity and pressure of the PDE, but the pulse length used for the initiation of the PDE can be an effective parameter to control the optimized performance and creation of detonation waves and propagation of the same for effective thrust production.

\section{References}

1. K. Kailasanath, AIAA paper 0470, (2002)

2. F. Falempin, Meeting Proceedings RTO-MP-AVT135, Paper 11. Neuilly-sur-Seine, France: RTO, (2006)

3. S.M. Frolov, J. Propul. Power, 22, 1162-1169 (2006)

4. W. Fan, C. Yan, X. Huang, et al., Combust. Flame 133 - 04, 441-450, (2003)

5. Z. Wang, C. Yan, W. Fan, L. Zheng Proc. Inst. Mech. Eng. G. J. Aerosp. Eng., 223, 721-728, (2009) doi:10.1243/09544100JAERO486.

6. Z. Wang, X. Chen, J. Huang, L. Zheng, C. Peng, Appl. Therm. Eng., 62, 407-414, (2014)

7. Z. Wang, J. Lu, J. Huang, C. Peng, L. Zheng, Appl. Therm. Eng., 73, 21-29, (2014)

8. C. Cathey, F. Wang, T. Tang, et al., AIAA paper, 443, (2007).

9. Z. Wang, X. Chen, L. Zheng, J. Lu, C. Peng, J. Propul. Technol., 35, 1434-1440, (in Chinese), (2014)

10. R. Knystautas, J.H.S. Lee, I.O. Moen, et al., The Combustion Institute, Pittsburgh, 1235-1244, (1979)

11. F. Carnasciali, J.H.S. Lee, R. Knystautas, Combust. Flame, 84, 170-180, (1991)

12. C.M. Brophy, L.T.S. Werner, J.O. Sinibaldi, AIAA paper, 84, 2003-1344, (2003)

13. C. Li, K. Kailasanath, J. Propul. Power, 21, 183186, (2005)

14. T. Yatsufusa, M. Ohira, S. Yamamoto, et al. AIAA paper 2004-1213, (2004)

15. S.P. Medvedev, S.V. Khomik, H. Olivier, et al., Shock Waves, 14, 193-203, (2005) 
16. H. Shimada, Y. Kenmoku, H. Sato, A.K. Hayashi, AIAA paper 2004-0308, (2004)

17. D. Bradley, C.G.W. Sheppard, I.M. Suardjaja, R. Woolley, Combust. Flame, 138, 55-77, (2004)

18. M. Li, C. Yan, J. Exp. Fluid Mech, 23, 92-97 (in Chinese), (2009)

19. J. Yu, Y. Qin, G. Gao, J. Aerosp. Power, 26, $1043-$ 1047 (in Chinese), (2011)

20. H. Zeng, L. He, X. Zhang, et al., J. Appl. Mech, 27, 543-548 (in Chinese), (2010)

21. Z. Wang, X. Chen, L. Zheng, et al., J. Propul. Technol., 34, 422-427 (in Chinese), (2013)

22. X. Cai, J. Liang, Z. Lin, R. Deiterding, F. Zhuang, Combust. Sci. Technol., 187, 525-536, (2015)

23. L. Zheng, C. Yan, W. Fan, et al., Journal of Combust. Sci. Technol., 9, 344-347 (in Chinese), (2003)

24. W. Chen, Q. Zhang, W. Fan, et al., J. Propul. Technol., 32, 296-300 (in Chinese), (2011)

25. G. Ciccarelli, S. Dorofeev, Prog. Energy Combust. Sci., 34, 499-550, (2008)

26. Z. Wang, C. Yan, L. Zheng, W. Fan, Combust. Sci. Technol., 181, 1310-1325, (2009)

27. S. Srikrishnan, Dr. P. K. Dash, IJMET, 8-6, 447454, (2017) 